\title{
Late Presentation of Aortic Aneurysm and Dissection Following Cardiac Catheterization
}

\author{
Abimbola Shofu ${ }^{\mathrm{a}}$, G. Mustafa Awan ${ }^{\mathrm{a}}$, Bassam Omar ${ }^{\mathrm{a}, \mathrm{b}}$, \\ Ghazanfar Qureshi ${ }^{\mathrm{a}}$
}

\begin{abstract}
We report a 63-year-old female with hypertension, hyperlipidemia, and prior pacemaker insertion for atrial fibrillation with symptomatic bradycardia, who was admitted with substernal chest pressure and diaphoresis. Her electrocardiogram revealed atrial fibrillation with demand ventricular pacing and her cardiac biomarkers were negative for acute coronary syndrome. Echocardiogram revealed normal left ventricular systolic function and normal aortic root diameter. Coronary angiography revealed $60-70 \%$ obtuse marginal lesion, otherwise mild disease. She was treated medically and discharged in stable condition. She was readmitted 1 month later with recurring chest pain, and shortness of breath which started shortly after her most recent discharge. Blood pressure was $152 / 93 \mathrm{~mm} \mathrm{Hg}$, and heart rate was 105 $\mathrm{bpm}$. BNP was elevated at $1,400 \mathrm{pg} / \mathrm{mL}$, and other cardiac biomarkers were negative. She was treated with diuretics, which resulted in decrease of her blood pressure to $81 / 51 \mathrm{~mm} \mathrm{Hg}$. Repeat echocardiogram revealed severely dilated aortic root, measuring $6.7 \mathrm{~cm}$, with aortic dissection flap and moderate to severe aortic regurgitation. CT angiogram revealed aortic dissection extending proximally to the aortic root above the coronary ostia and distally to the left subclavian artery takeoff. She underwent surgery; she, however, could not be weaned off from cardiopulmonary bypass and died in the operating room. This case illustrates the importance of having a high index of suspicion for iatrogenic aortic dissection following cardiac catheterization as a cause of recurrence of cardiac symptoms, as early detection may help avert a catastrophic outcome, as we report in our patient.
\end{abstract}

Keywords: Cardiac catheterization; Aortic dissection; Coronary disease; Complications

\section{Introduction}

Aortic dissection following coronary angiography and an-

Manuscript accepted for publication March 08, 2017

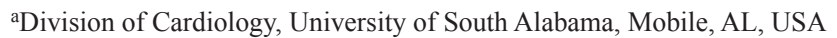
${ }^{\mathrm{b} C o r r e s p o n d i n g ~ A u t h o r: ~ B a s s a m ~ O m a r, ~ D i v i s i o n ~ o f ~ C a r d i o l o g y, ~ U n i v e r s i t y ~ o f ~}$ South Alabama, Mobile, AL 36617, USA.

Email: omarbassamanwar@gmail.com

doi: https://doi.org/10.14740/cr537w gioplasty is a rare, but potentially fatal complication with an overall reported incidence of $0.01-0.04 \%$ [1-3]. The incidence is significantly higher during percutaneous coronary interventions $(0.12 \%)$ than during elective diagnostic procedures [1]. Moreover, in the setting of acute myocardial infarction (AMI), the reported incidence is $0.19 \%$, significantly higher than the $0.01 \%$ reported in the absence of AMI [2]. Management and outcomes have varied considerably in the literature. While up to $50 \%$ mortality was reported in one series following surgical repair of the dissections [3], other studies reported more favorable outcomes with conservative management.

We report a case of an elderly woman who developed type A aortic dissection with moderate to severe aortic regurgitation and heart failure, diagnosed approximately 1 month following a diagnostic coronary angiography. She underwent emergency surgical repair of the dissection; however, she could not be successfully weaned off of cardiopulmonary bypass despite maximum pharmacological interventions and support and died in the operating room.

\section{Case Report}

A 63-year-old white female with known hypertension, hyperlipidemia, and prior pacemaker insertion for atrial fibrillation with symptomatic bradycardia, was admitted to our hospital with recent onset substernal chest pressure radiating to her jaw, with diaphoresis. Her vital signs were stable, and her physical examination was non-revealing, but was limited by her morbid obesity. Her electrocardiogram revealed atrial fibrillation with demand ventricular pacing and her cardiac biomarkers were negative for acute coronary syndrome. Echocardiogram revealed normal left ventricular systolic function and normal aortic root diameter, otherwise was technically difficult (Fig. 1a). Coronary angiography revealed $60-70 \%$ eccentric lesion in the second obtuse marginal, otherwise mild atherosclerotic disease. She was treated medically and later discharged in stable condition.

She was readmitted 1 month later with recurring episodes of chest pain, shortness of breath, orthopnea and reduced exercise tolerance which started ever since her recent discharge, for which she had two emergency room visits and a clinic followup visit. Blood pressure (BP) was 152/93 $\mathrm{mm} \mathrm{Hg}$, and heart rate was $105 \mathrm{bpm}$. Physical examination was again limited by her body habitus. She had an elevated BNP of 1,400 pg/mL, but otherwise negative cardiac biomarkers, and was therefore treated with diuretics, which resulted in a significant decrease of her 

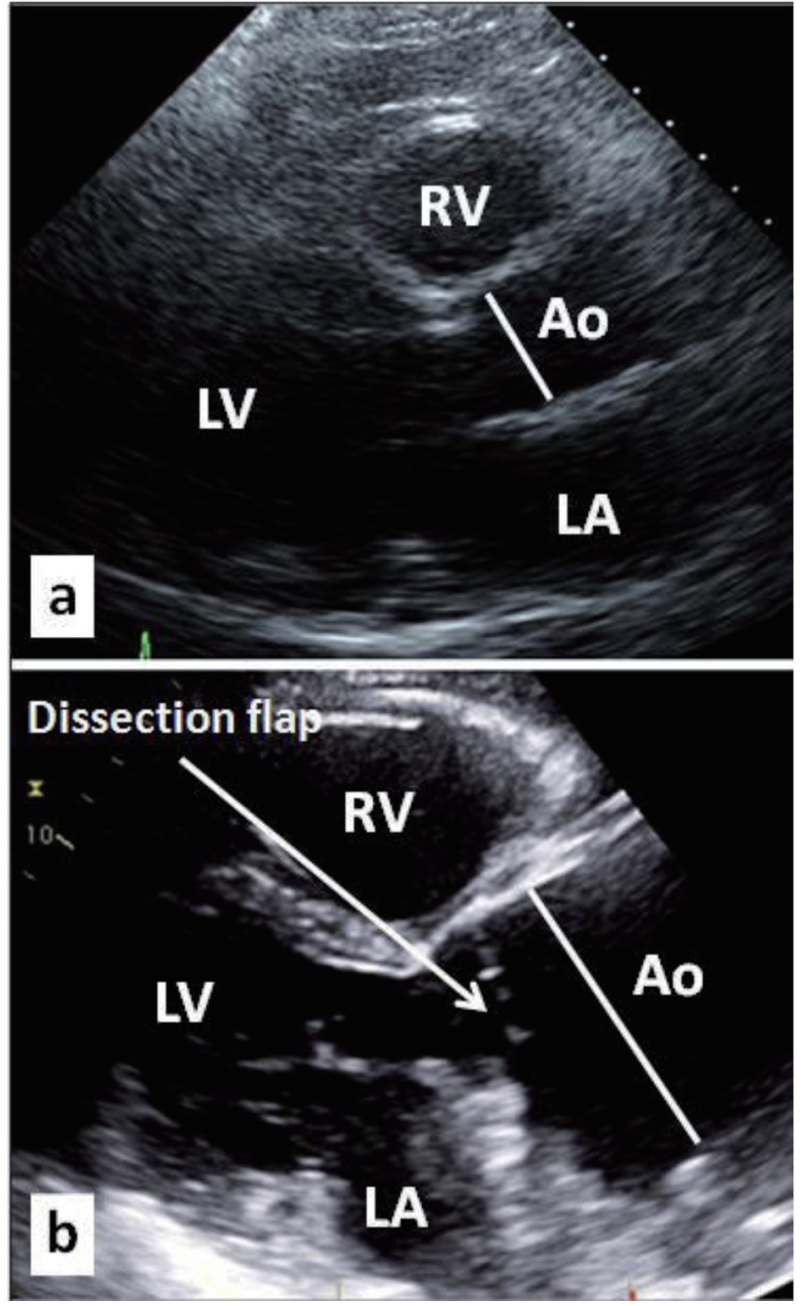

Figure 1. (a) Baseline two-dimensional (2D) parasternal long axis echocardiographic image obtained prior to index cardiac catheterization showing normal size aortic root and ascending aorta (Ao). (b) Corresponding 2D parasternal echocardiographic image 1 month following cardiac catheterization showing massive dilatation of the aortic root and ascending aorta (Ao) with a dissection flap. LV: left ventricle; RV: right ventricle; LA: left atrium.

BP to $81 / 51 \mathrm{~mm} \mathrm{Hg}$. Repeat echocardiogram was performed, which revealed normal left ventricular systolic function, and severely dilated aortic root, measuring $6.7 \mathrm{~cm}$, with aortic dissection flap noted (Fig. 1b) and moderate to severe aortic regurgitation. CT angiogram revealed aortic dissection extending proximally to the aortic root above the coronary ostia (Fig. 2a) and distally to the left subclavian artery takeoff (Fig. 2b).

The patient was taken emergently to surgery and despite extensive surgical repair and pharmacologic support, she could not be weaned off of cardiopulmonary bypass and expired in the operating room.

\section{Discussion}

Type A aortic dissection is a lethal condition with an overall
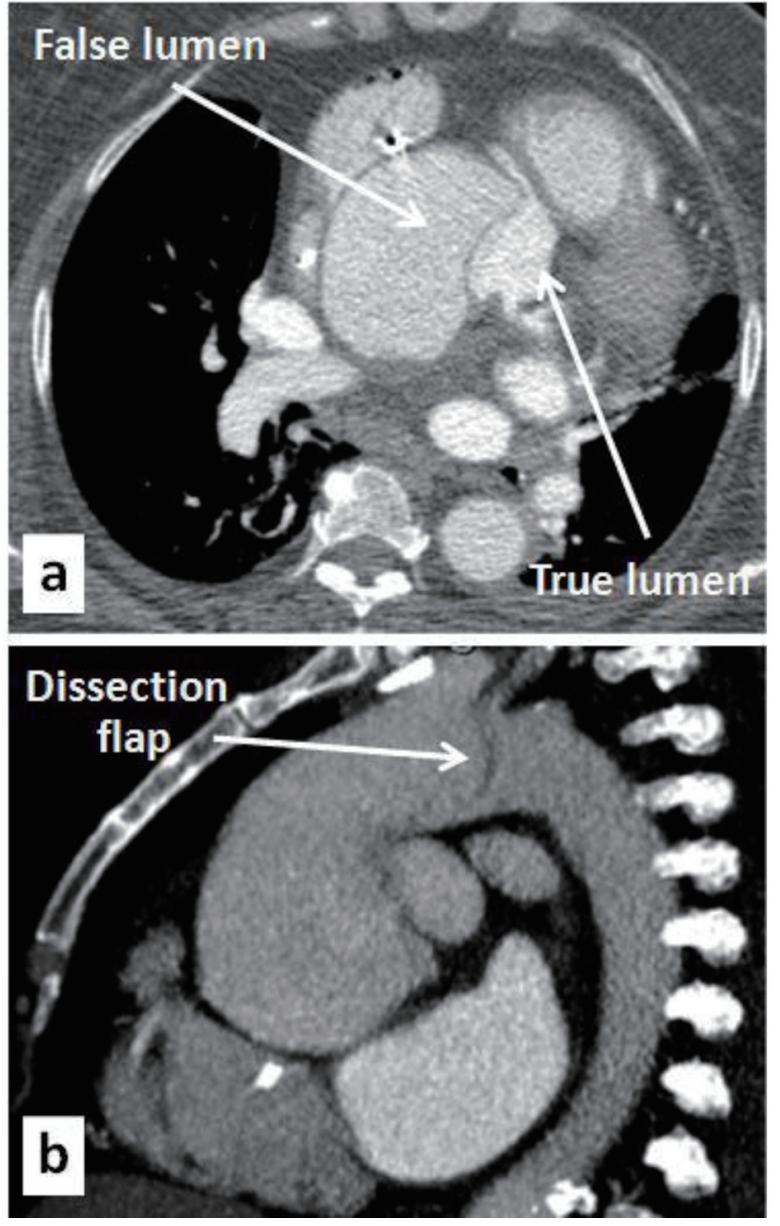

Figure 2. (a) Axial CT angiographic image showing the dissection starting at the aortic root and sparing the origins of the coronary arteries, with massive dilatation of the aortic root. (b) Sagittal CT angiographic image showing the extension of the dissection to the origin of the brachiocephalic artery.

surgical mortality of up to $34 \%$ in unstable patients, as reported [4] in 526 cases by the International Registry of Acute Aortic Dissection (IRAD). The IRAD registry reported 28 cases $(5.3 \%)$ of iatrogenic type A aortic dissection (ITAAD) as a complication of cardiac surgery or cardiac catheterization, nine of which $(32 \%)$ died. The German Registry for Acute Aortic Dissection Type A (GERAADA) reported a comparable incidence of 100 cases $(4.7 \%)$ of ITAAD out of a total of 2,137 cases of type A aortic dissection, but with a lower 30-day mortality of $16 \%$ [5].

ITAAD remains a rare complication of coronary angiography and open heart surgery. Leontyev et al [1] reported 48 cases over a 15-year follow-up, undergoing surgery for ITAAD, with a frequency of $0.06 \%$ (36 cases) of open heart surgeries, and $0.01 \%$ (12 cases) of cardiac catheterizations; early surgical mortality was $50 \%$ for ITAADs associated with coronary angiography. Dunning et al [2] reported nine cases $(0.02 \%)$ of coronary artery - aortic dissections out of over 40,000 cardiac catheterizations performed over a 6-year period; these were significantly more prevalent in the setting of AMI $(0.19 \%)$, 
Table 1. Summary of ITAAD Case Reports With Favorable Outcomes

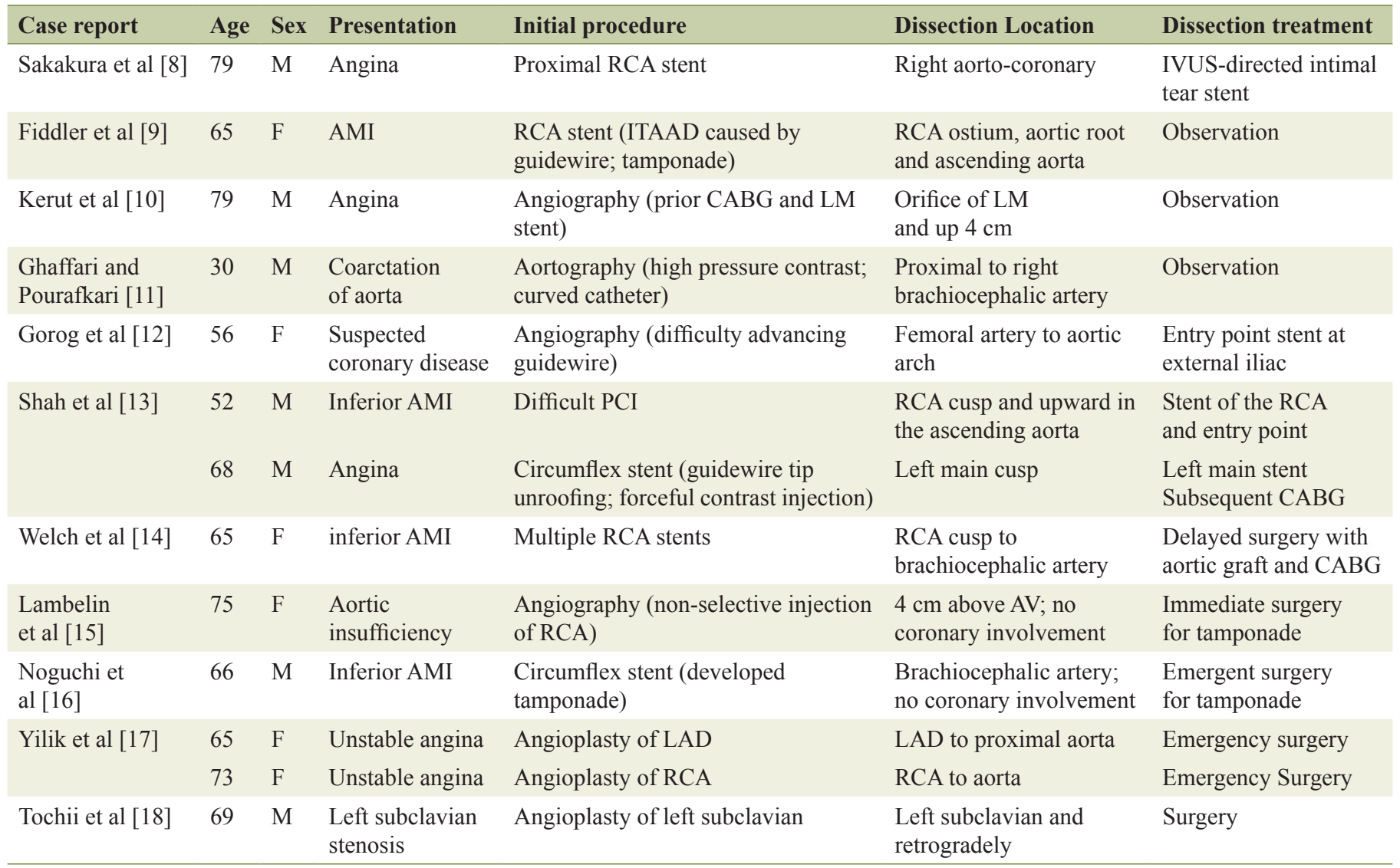

compared with non-AMI $(0.01 \%)$. While outcomes were favorable for the less severe dissections treated conservatively, two of the reported cases had dissection extending into the arch, and both died following surgery. They proposed a classification scheme based on the extent of aortic dissection beyond the involved coronary cusp (Fig. 3 [2]), and concluded that the best treatment in class 1 and 2 dissections is stenting of the intra-coronary entry point when possible and close clinical follow-up, while class 3 dissections usually require surgical intervention. Gomez-Moreno et al [3] reported 17 cases $(0.04 \%$ incidence) of ITAAD associated with cardiac catheterizations over a 10-year follow-up period, with a significantly higher incidence after interventional procedures $(0.12 \%)$ than after diagnostic procedures $(0.01 \%)$. Patients were treated conservatively with either stenting to seal the entry door or expectant management; no patients died during hospitalization or followup. Nunez-Gil et al [6] reported 14 cases $(0.02 \%$ incidence) of iatrogenic dissection of the descending aorta/arch without coronary involvement over a 15-year follow-up treated conservatively, with only one hospital death. Tanasie et al [7] reported eight cases over a 5-year follow-up of ITAADs referred for multi-detector coronary tomography (MDCT) evaluation; one patient required surgery and died, while all others treated conservatively with either stenting or expectant management survived.

Several cases of conservatively treated ITAAD have been reported in the literature with favorable outcomes. Sakakura et al [8] reported a 79-year-old male with ITAAD following stenting of proximal right coronary artery (RCA) for angina who was treated with intravascular ultrasound (IVUS)-guided stenting of the entry point with good outcome. Fiddler et al [9] reported a 65-year-old female who underwent RCA stenting for AMI resulting in guidewire-associated ITAAD with evidence of tamponade; there was spontaneous hemodynamic stabilization and subsequent CT demonstrating no intimal dissection flap, prompting expectant management with favorable outcome. Kerut et al [10] reported a 79-year-old male undergoing coronary angiography for angina, who developed subintimal hematoma, without dissection flap, in the left coronary sinus and $4 \mathrm{~cm}$ upwards as demonstrated by $\mathrm{CT}$ and transesophageal echocardiography (TEE); these findings resolved spontaneously and she was treated conservatively with favorable outcome. Ghaffari and Pourafkari [11] reported ITAAD proximal to the origin of the right brachiocephalic artery in a 30-year-old male with bicuspid aortic valve undergoing aortography in preparation for aortic coarctation stenting. The dissection spontaneously sealed, and they attributed this to curved catheter position in the aortic arch and high-pressure contrast jet through the catheter side holes in the setting of aortopathy. Gorog et al [12] reported a 56-year-old female undergoing coronary angiography who developed aortic dissection starting at the femoral artery extending into the aortic arch, treated successfully 


\begin{tabular}{lll}
\hline Class & $\begin{array}{l}\text { Coronary cups/aortic } \\
\text { involvement }\end{array}$ & $\begin{array}{l}\text { Proposed } \\
\text { treatment }\end{array}$ \\
\hline I & Ipsilateral cusp only & $\begin{array}{l}\text { Stenting of the } \\
\text { intra-coronary entry } \\
\text { point and close } \\
\text { clinical follow-up }\end{array}$ \\
II $\quad$ Extending $<4.0 \mathrm{~cm}$ up the aorta
\end{tabular}

Figure 3. Dunning classification of ITAAD [2].

with a self expanding metallic stent into the iliac artery via the contralateral femoral artery approach. Shah et al [13] reported two cases of ITAAD, the first was a 56-year-old male receiving RCA stent for inferior AMI who developed ITAAD starting at the RCA cusp, successfully treated with stenting of the proximal RCA to cover the entry point. The second case was a 68-year-old male who developed left main coronary dissection with retrograde extension into the coronary cusp during stenting of the left circumflex, treated successfully with left main stenting; he subsequently underwent an uneventful elective coronary artery bypass grafting (CABG) surgery.

Surgically treated ITAAD cases have also been reported in the literature with successful outcomes. Welch et al [14] reported a 65-year-old female treated with multiple RCA stents for inferior AMI who developed an "intramural hematoma" extending from the right sinus of Valsalva to the origin of the brachiocephalic artery, with an entry point. She was treated surgically after a period of stabilization and recovered well. Lambelin et al [15] reported a 75-year-old female who developed cardiac tamponade and cardiogenic shock due to intimal tear in the ascending thoracic aorta, not involving the coronaries, during angiography for aortic insufficiency; she was successfully treated with immediate surgery. Noguchi et al [16] reported a 66-year-old male with inferior AMI undergoing circumflex stenting, who developed cardiac tamponade and hypotension due to ITAAD originating at the brachiocephalic artery, without involvement of the coronaries; he underwent successful emergency surgical repair. Yilik et al [17] reported two cases of ITAAD treated surgically, the first was a 65 -yearold female who developed retrograde aortic dissection during angioplasty of the left anterior descending (LAD) for unstable angina; due to inability to localize the tear, she underwent uneventful surgical repair. The second case was a 73-year-old female who developed ITAAD during angioplasty to the RCA, the guidewire could not be introduced into the true lumen and therefore she underwent successful surgical repair. Tochii et al [18] reported a 69-year-old male who developed aortic dissection during balloon angioplasty to the left subclavian which extended retrogradely to the ascending aorta and required surgical intervention with good outcome.

The above case reports, summarized in Table 1 [8-18], demonstrate the heterogeneity of ITAAD with regard to patient characteristics and presentations, cause and location of the dissection, and treatment options. No guidelines exist as how to approach these complications. It is obvious that severe dissections are associated with high early mortality and therefore warrant emergency surgery, which in itself carries very high risk of mortality. Our patient is unique in that her presentation was delayed at 1 month from her index cardiac catheterization, with progressive symptoms, likely causing extensive aortic root dilatation and extension of dissection (Dunning class III). Furthermore, her hemodynamic instability necessitated emergency surgery, which she could not tolerate. Negative outcomes may in fact be more prevalent than our discussion implies, but may tend to be under-reported in the literature.

\section{Conclusion}

Aortic dissections after coronary angiography are rare but can be fatal. Associated risk factors reported in several studies may be related to patient characteristics versus procedural variables, some of which may be modifiable (Table 2). Prompt recognition and management is crucial to avoid the extensive dissection and dilatation of the aorta, as we report in our patient, and reduce mortality. Low risk dissections appear to respond well to conservative medical and localized interventional treatments, with serial hemodynamic monitoring, im-

Table 2. Potential Patient Characteristics and Procedural Variables Associated With ITAAD

\begin{tabular}{ll}
\hline Patient characteristics & Procedural variables \\
\hline AMI & PCI (balloon, stent) \\
Unstable angina & High pressure contrast injection \\
PCI/CABG history & Nonselective coronary injection \\
Aortopathy & Guidewire unroofing \\
Atherosclerosis & Curved catheter \\
Male & Difficult guidewire maneuver \\
Elderly & RCA involvement \\
\hline
\end{tabular}


aging and follow-up. High risk dissections overall appear to require surgical management, despite the increased surgical risk.

\section{Disclosures}

All authors declare no disclosures related to this manuscript.

\section{References}

1. Leontyev S, Borger MA, Legare JF, Merk D, Hahn J, Seeburger J, Lehmann S, et al. Iatrogenic type A aortic dissection during cardiac procedures: early and late outcome in 48 patients. Eur J Cardiothorac Surg. 2012;41(3):641646.

2. Dunning DW, Kahn JK, Hawkins ET, O'Neill WW. Iatrogenic coronary artery dissections extending into and involving the aortic root. Catheter Cardiovasc Interv. 2000;51(4):387-393.

3. Gomez-Moreno S, Sabate M, Jimenez-Quevedo P, Vazquez P, Alfonso F, Angiolillo DJ, Hernandez-Antolin $\mathrm{R}$, et al. Iatrogenic dissection of the ascending aorta following heart catheterisation: incidence, management and outcome. EuroIntervention. 2006;2(2):197-202.

4. Trimarchi S, Nienaber CA, Rampoldi V, Myrmel T, Suzuki T, Mehta RH, Bossone E, et al. Contemporary results of surgery in acute type A aortic dissection: The International Registry of Acute Aortic Dissection experience. J Thorac Cardiovasc Surg. 2005;129(1):112-122.

5. Rylski B, Hoffmann I, Beyersdorf F, Suedkamp M, Siepe M, Nitsch B, Blettner M, et al. Iatrogenic acute aortic dissection type A: insight from the German Registry for Acute Aortic Dissection Type A (GERAADA). Eur J Cardiothorac Surg. 2013;44(2):353-359; discussion 359.

6. Nunez-Gil IJ, Bautista D, Perez-Vizcaino MJ, Cerrato E, Salinas P, Fernandez-Ortiz A. Type A iatrogenic aortic dissection following catheterization without coronary involvement: long-term prognosis. Rev Esp Cardiol (Engl Ed). 2015;68(3):254-255.

7. Tanasie C, Chandonnet M, Chin A, Kokis A, Ly H, Perrault LP, Chartrand-Lefebvre C. Catheter-induced aortic dissection after invasive coronary angiography: evaluation with
MDCT. AJR Am J Roentgenol. 2011;197(6):1335-1340.

8. Sakakura K, Wada H, Taniguchi Y, Mori M, Momomura $\mathrm{S}$, Ako J. Intravascular ultrasound-guided coronary stenting without contrast medium for the treatment of catheter-induced aortocoronary dissection. Cardiovasc Interv Ther. 2013;28(1):71-75.

9. Fiddler M, Avadhani SA, Marmur JD. Guide catheterinduced aortic dissection complicated by pericardial effusion with pulsus paradoxus: a case report of successful medical management. Case Rep Med. 2015;2015:480242.

10. Kerut EK, Falterman C, Hanawalt C, Frank R, Everson $\mathrm{C}$, Hunter D. Iatrogenic localized ascending aortic intimal hematoma during left coronary artery catheterization. Echocardiography. 2005;22(9):785-787.

11. Ghaffari S, Pourafkari L. Images in clinical medicine. Aortic dissection during diagnostic aortography. N Engl J Med. 2010;363(13):e18.

12. Gorog DA, Watkinson A, Lipkin DP. Treatment of iatrogenic aortic dissection by percutaneous stent placement. J Invasive Cardiol. 2003;15(2):84-85.

13. Shah P, Bajaj S, Shamoon F. Aortic Dissection Caused by Percutaneous Coronary Intervention: 2 New Case Reports and Detailed Analysis of 86 Previous Cases. Tex Heart Inst J. 2016;43(1):52-60.

14. Welch TD, Foley T, Barsness GW, Spittell PC, Tilbury RT, Enriquez-Sarano M, Evangelista A, et al. Iatrogenic aortic dissection... or intramural hematoma? Circulation. 2012;125(9):e415-418.

15. Lambelin M, Janssens L, Haenen L. Iatrogenic Ascending Aorta Dissection during Diagnostic Coronary Angiography: Rare but Life-Threatening. Case Rep Cardiol. 2014;2014:809398.

16. Noguchi K, Hori D, Nomura Y, Tanaka H. Iatrogenic Acute Aortic Dissection during Percutaneous Coronary Intervention for Acute Myocardial Infarction. Ann Vasc Dis. 2012;5(1):78-81.

17. Levent Yilik, Banu Lafci, Ibrahim Ozsoyler, et al. Surgical treatment of aortic dissection occurring during coronary angioplasty: a report of two cases. Turkish J Thorac Cardiovasc Surg. 2007;15(3):238-241.

18. Tochii M, Ando M, Takagi Y, Kaneko K, Ishida M, Akita $\mathrm{K}$, Higuchi Y. Iatrogenic type A aortic dissection after catheter intervention for the left subclavian artery. Ann Thorac Cardiovasc Surg. 2010;16(6):451-453. 